through the tail of Giacobini-Zinner in September 1985 and a five-spacecraft "fleet" arrives at comet Halley in March, 1986.

In December 1983 the project scientists for each comet space mission very graciously gave their time to present invited talks to AGU members at the AGU 1983 Fall Meeting in San Francisco, Calif. T. I. Gombosi (Hungarian Academy of Sciences) presented the dual VEGA (acronym of "Venera-Halley" in Russian) mission and expected results, $\mathbf{R}$. Reinhard (ESA) presented the Giotto mission, H. Oya (Tohoku University) presented the Japanese MS-T5 and Planet-A missions, and T. T. von Rosenvinge (Goddard Space Flight Center) presented the ICE mission. Each project scientist kindly agreed to write up his talk for the Eos readership. With the launch of the Soviet VEGA 1 on December 15, 1984, it was thought that it would be appropriate and timely to publish Tamas Gombosi's article on the VEGA mission now. This mission, as will be evident, is one of the most ambitious remote-controlled investigations ever launched into space.

\section{Acknowledgments}

Discussions with B. E. Goldstein, M. Neugebauer, R. L. Newburn, and E. J. Smith were helpful and are appreciated. This report represents one aspect of research carried out by the Jet Propulsion Laboratory, California Institute of Technology, for NASA.

\section{References}

Alfvén, H., On the theory of comet tails, Tellus, 9, 92, 1957.

Houpis, H. L. F., and D. A. Mendis, Physiochemical and dynamical processes in cometary ionospheres, 1, The basic flow profile, Astrophys. J., 239, 1107, 1980.

Ip, W.-H., and W. I. Axford, Theories of physical processes in the cometary comae and ion tails, in Comets, edited by L. Wilkening, p. 588, University of Arizona Press, Tucson, 1982.

Oort, J. H., The structure of the cometary cloud surrounding the solar system and a hypothesis concerning its origin, Bull. Astron. Inst. Neth., 11, 91, 1950.

Wallis, M. K., and M. Dryer, Sun and comets as sources in an external flow, Astrophys. J., 205, 895, 1976.

Whipple, F. L., A comet model, 1, The acceleration of comet Encke, Astrophys. J., 111, $375,1950$.

\section{Suggested Reading}

Bird, M. K. (ed.), Observations of Comets: Radio and Otherwise, Max Planck Inst. Radioastron. Tech. Ber. 64, 1984.

Breus, T. K., Solar wind interactions with comets, Space Sci. Rev., 32, 361, 1982.

Cameron, A.G.W., Accumulation processes in the primitive solar nebula, Icarus, 18, 407, 1973.

Gombosi, T. I. (ed.), Cometary Exploration, Central Research Institute for Physics, Hungarian Academy of Sciences, Budapest, 1982.

Mendis, D. A., and H. L. F. Houpis, The cometary atmosphere and its interaction with the solar wind, Rev. Geophys. Space Phys., 20, 885, 1982.

Neugebauer, M., D. K. Yeomans, J. C. Brandt, and R. W. Hobbs, Space missions to comets, NASA Conf. Publ. 2089, 1979.

Smith, E. J., et al., Intercept of Giacobini-Zinner by ISEE 3, report, Goddard Space Flight Ctr., Greenbelt, Md., 1983.

Wilkening, L. L., Comets, University of Arizona Press, Tucson, 1982.

\title{
VEGA: En Route to Venus and Comet Halley
}

\section{Tamas I. Gombosi ${ }^{1}$}

Space Physics Research Laboratory, University of Michigan, Ann Arbor

PAGES 33, 35-36

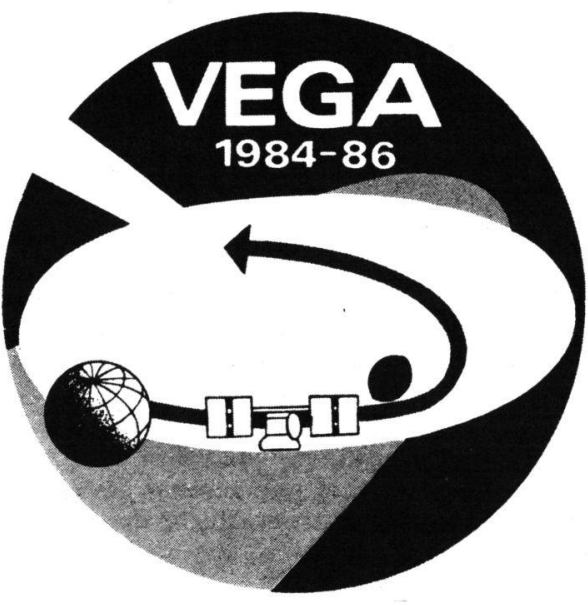

On December 15 and 21, 1984, the Soviet Union launched VEGA 1 , followed by an almost identical twin, VEGA 2. VEGA (the name is an acronym of "Venus-Halley," the two targets of the space probes, in Russian) is the first deep space mission to carry a truly

${ }^{1}$ Permanent address: Central Research Institute for Physics, Hungarian Academy of Sciences, Budapest. international scientific payload. In addition to the Soviets, who are bearing most of the costs, France and Hungary also played a major role in putting together the complex of instruments. Scientists from Austria, Bulgaria, Czechoslovakia, the Federal Republic of Germany and the German Democratic Republic, Poland, and the United States also have contributed to this very ambitious program. U.S. scientists participate on an individual basis in VEGA: Thomas Cravens and Andrew Nagy of the University of Michigan take part in the particle science team; D. Asoka Mendis of the University of California, San Diego, participates in the spectroscopic team; and Bradford Smith of the University of Arizona is a member of the television science group. John Hsieh of the University of Arizona and John Simpson of the University of Chicago designed detectors for neutral gas and dust, respectively, made jointly with scientists of the Max Planck Society, Federal Re public of Germany (FRG).

The generally accepted view of the scientific community is that comets were formed together with the rest of the solar system approximately 4.5 billion years ago. These estimated million million $\left(10^{12}\right)$ planetesimals were created in the outer, cooler part of the presolar nebula. They did not form into the giant planets but reached the outer edge of the planetary system to form the so-called Oort cloud. Because of the effect of gravitational perturbations, some of these deep-frozen dust-ice conglomerates leave the Oort cloud and can propagate toward the inner part of the solar system. Not all of these planetesimals make it to the proximity of the sun: the majority only get to "parking" orbits whose perihelia are beyond the orbits of the giant planets Jupiter and Saturn. It is from these parking orbits that the spectacular active comets originate. With the help of gravitational perturbations caused by the giant planets, the cometary bodies find their way to final orbits approaching the sun.

When one of these dirty frozen snowballs approaches the sun, its surface gradually warms up, and at a distance of about $3 \mathrm{AU}$, carbon monoxide starts to sublimate. When the comet gets closer than about 1.5 AU to the sun, water vapor becomes the dominant sublimating species. The freshly sublimated gas leaves the surface at slightly less than the local speed of sound, and it propagates as a "cometary wind" into interplanetary space. The sublimating gases are capable of blowing away specks of dust from the surface. Solar radiation and other effects partially ionize the outflowing gas, which then interacts strongly with the solar wind. Radiation pressure and solar gravity act on the dust grains. It is these effects that cause the cometary coma and the tail

When they arrive at Venus in June 1985 , the two VEGAs will each release a $1500-\mathrm{kg}$ entry probe for a nightside landing on the Venusian surface. About $55 \mathrm{~km}$ above the unfriendly surface (the surface pressure is about 100 times that of the earth, and the temperature is about 25 times greater) the descending probes will each dispatch a balloon for tracking wind velocities.

After release of their Venus probes the two modified Venera-class, three-axis stabilized spacecraft (see Figure 1) will continue their voyage toward their Halley encounters. The planned orbit is shown in Figure 2. The probes will pass the comet at a distance of about $10,000 \mathrm{~km}$ on the sunward side at about 0300 UT on March 6 and 9, respectively. At the encounter, the spacecraft will have 


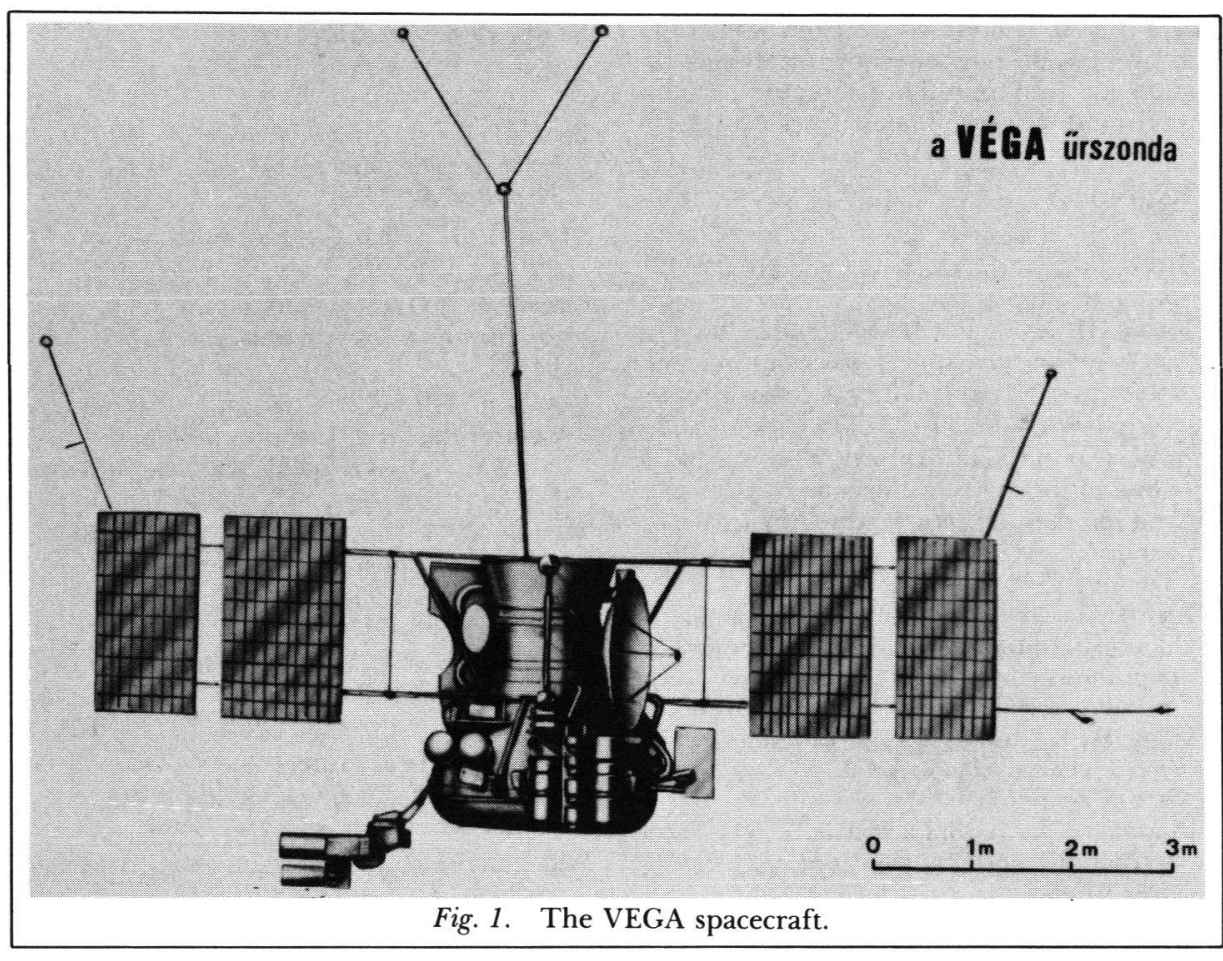

a velocity of $78 \mathrm{~km} / \mathrm{s}$ with respect to the comet.

Figure 3 shows the encounter geometry. The $z$ axis points towards the sun, while $x$ is perpendicular to the ecliptic plane, pointing northward. In order to see the center of the coma at a preencounter point (marked $\mathrm{A}$ in Figure 3), all optical instruments have to look ahead, along the $s / c$ trajectory, and somewhat upward. After encounter (point $\mathbf{C}$ in Figure 3) the instruments have to look back and downward.

Since the spacecraft is three-axis stabilized, a rotating pointing platform will ensure that the nucleus, never before seen, remains in the field of view of the imaging instruments. This platform has to move with an angular velocity of about $1 \%$ near closest approach $\left(B_{c}\right.$ in Figure 3). The field of view of the narrow-angle main television camera is only about $0.5^{\circ}$; thus there is a high degree of onboard tracking accuracy required to lock the camera onto the nucleus. The crucial final phase of the encounter lasts only about 5-10 minutes. Because this occurs at an approximate distance of $1 \mathrm{AU}$ from the earth, it is not possible to use remote control. From the images of the television camera, a built-in onboard computer system has to be able to recognize the cometary nucleus and command the pointing platform to track it at the same time that the spacecraft speeds past the central part of the optically thick coma.

Figure 4 illustrates the TV camera and its interface with the rotating platform. Three of the four charge-coupled devices (CCD's) work in a photographic regime to provide a processed picture every few seconds. On the basis of subsequent pictures the central microprocessor of the "pointing/predicting" unit continuously updates orbital elements for the nucleus and predicts its position a few seconds ahead. The TV/platform interface unit directs the platform rotation around its two axes based on these predictions. The fourth CCD operates in a mode different from the rest. It continuously provides $\sim 10-\mathrm{Hz}$ frequency TV pictures of the nucleus (commercial TV pictures use a $25-\mathrm{Hz}$ frequency). These pictures are analyzed in real time by a separate electronic unit which is able to give platform control commands after having processed each frame. This command line is used as one of several backups to be activated under certain circumstances.

At comet Halley the VEGA probes will have the following scientific objectives:

- To determine the physical characteristics and chemical structure of the nucleus;

- To identify the parent molecules of the coma (gas molecules leaving the surface)

- To determine the chemical composition and size distribution of the dust particles at different distances from the nucleus;

- To determine the chemical composition of the coma at different distances from the nucleus;

- To investigate the interaction between the solar wind and the cometary atmosphere and ionosphere.

To meet these objectives, the probe has been equipped with the scientific instruments whose descriptions follow.

\section{TV System}

This instrumentation consists of two TV cameras and several on-board microprocessors. The instruments will obtain images in various spectral bands and in white light from different areas in the vicinity of the center of the coma. The optical systems of the cameras have focal lengths of 150 and 1200 $\mathrm{mm}$, resulting in view angles of $3.5^{\circ} \times 5.2^{\circ}$ and $0.4^{\circ} \times 0.6^{\circ}$, respectively, and $512 \times 576$ CCD light-sensitive matrices are used. For a comet-space probe distance of $10,000 \mathrm{~km}$ the resolution of the narrow angle camera on the surface of the cometary nucleus is $180 \mathrm{~m}$.

The TV system is a French, Hungarian, and Soviet joint experiment.

\section{Three-Channel Spectrometer}

This instrument will measure the light of the comet in the UV $(120-350 \mathrm{~nm})$, visible $(350-900 \mathrm{~nm})$, and IR $(900-2000 \mathrm{~nm})$ wavelength bands. The instrument is designed to

- Study the spectrum of the nucleus and of the region around the nucleus;

- Determine the parent molecules;

- Investigate the spectrum and polarization of the light scattered by the dust;

- Analyze the chemical composition of the coma;

- Map the coma;

- Measure the velocity of the different gas molecules.

The spectrometer is a joint venture of $\mathrm{Bu}$ garia, France, and the Soviet Union.

\section{Infrared Spectrometer}

This instrument comprises three optical channels, two of which serve for investigations in the 4000-8000-nm and 8000-16000 $\mathrm{nm}$ wavelength ranges, respectively. The third channel, operating in the 7000-14000$\mathrm{nm}$ range, will produce an infrared image of the nucleus. This spectrometer will help to determine the following:

- Dimension, radiation intensity and temperature of the nucleus;

- Composition, density distribution, and temperature of the dust particles;

- Distribution of the parent molecules and their density and velocity relative to a reference parent molecule.

The infrared spectrometer was produced by French experts

\section{Dust Mass Spectrometer}

This instrument will be used to determine the mass and chemical composition of the dust particles which form a cloud around the cometary nucleus. Dust particles evaporate upon hitting a silver plate, and some fraction becomes ionized. The number and mass of the ions thus created are measured. The values obtained are assumed to be representative of the mass and composition of the primary particle. The instrument is suitable for analyzing dust particles with masses between $3 \times 10^{-16}$ and $5 \times 10^{-10} \mathrm{~g}$. This equipment is a cooperative project of the FRG, France, and the Soviet Union.

\section{Dust Impact Counters}

Three types of dust impact counters are employed on the VEGA probes.

The first, an acoustic counter, consists of several metal plates stacked in series. Each plate is equipped with three piezoelectric sensors which will detect acoustic waves. The impact of a dust particle induces vibrations in the metal plate, and these vibrations are evaluated by the piezoelements. From the amplitude of the vibrations and from the time delay between individual detectors the impact can be located precisely, and the momentum of the dust particle can be determined. In addition, the mass of a particle is obtained with knowledge of the relative velocity of about 78 $\mathrm{km} / \mathrm{s}$. The instrument, oriented in the direction of the relative velocity vector of the spacecraft and the comet, is able to detect particles above a mass of $10^{-10} \mathrm{~g}$.

The second, a dust particle counter, is an instrument that counts the plasma clouds cre- 

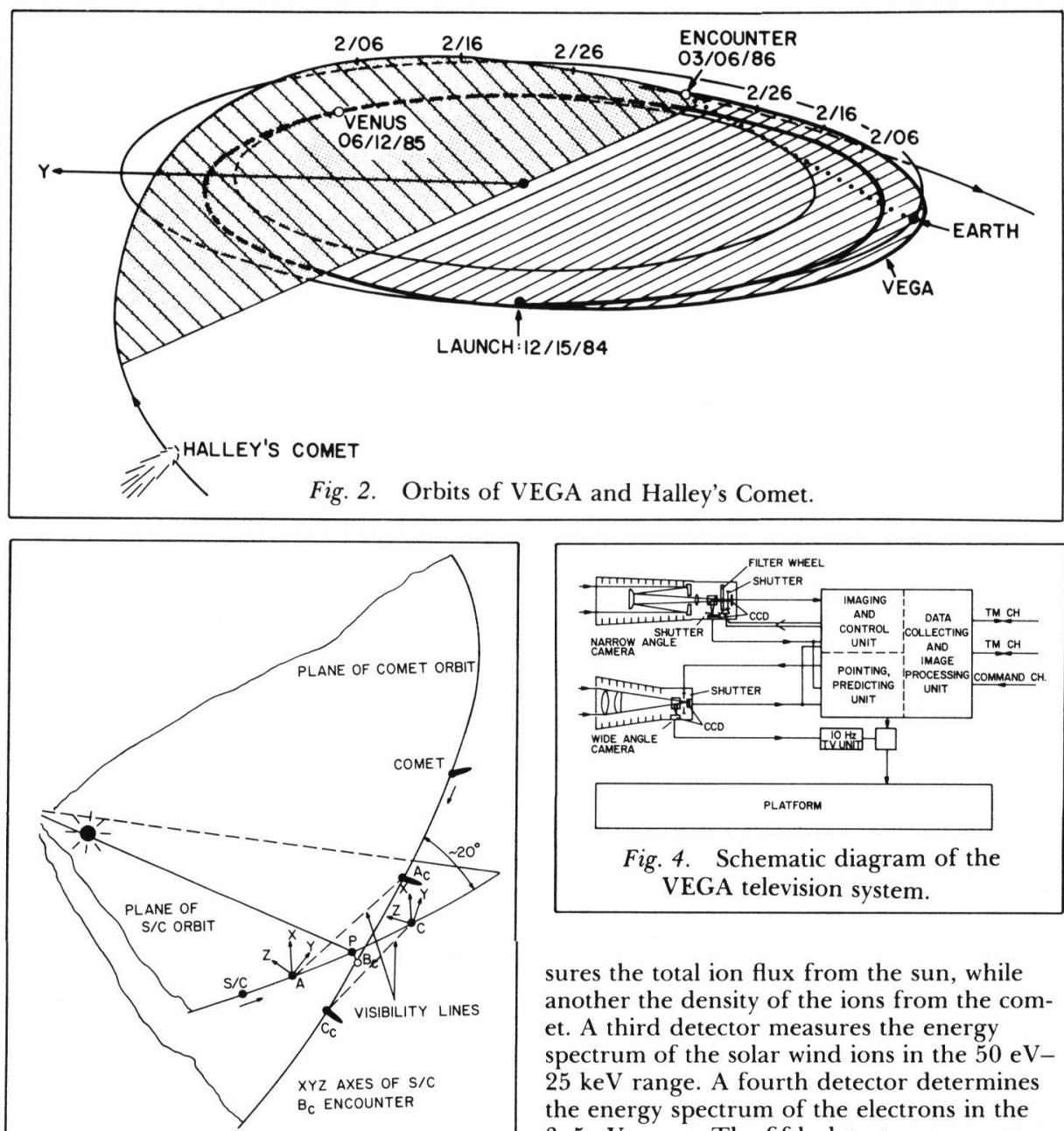

Fig. 3. VEGA encounter geometry.

ated by evaporating dust particles in the $10^{-12}$ to $10^{-18} \mathrm{~g}$ range. Both of these dust counters were made in the Soviet Union. The third, a solid-state dust detector, was made by University of Chicago scientists in cooperation with the Max-Planck-Institut für Aeronomie, FRG.

\section{Neutral Gas Mass Spectrometer}

Although the principle of operation of this instrument is very simple in a technical sense, its implementation is very difficult. The neutral gas molecules incident on the spectrometer are first ionized, and then the ions obtained are accelerated to a known energy. A very small time-of-flight analyzer (about 10 $\mathrm{cm}$ in size) measures the velocity of the ions. From knowledge of the ion energies and velocities, the masses can easily be calculated. This instrument represents a HungarianFRG-Soviet-U.S. cooperative effort.

\section{Ion and Electron Spectrometer}

This instrument consists of five detectors and their electronic units. One detector mea-

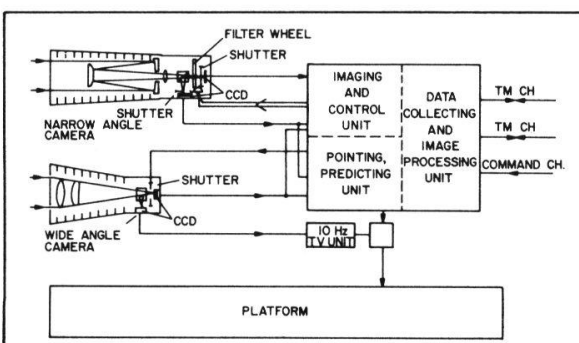

Fig. 4. Schematic diagram of the VEGA television system.

sures the total ion flux from the sun, while another the density of the ions from the comet. A third detector measures the energy spectrum of the solar wind ions in the $50 \mathrm{eV}$ $25 \mathrm{keV}$ range. A fourth detector determines the energy spectrum of the electrons in the $3-5-\mathrm{eV}$ range. The fifth detector measures the energy spectrum of the cometary ions. The latter information will be used to determine the ion mass spectrum by taking into account the relative velocity of $78 \mathrm{~km} / \mathrm{s}$. This instrument is the product of Hungarian-Soviet cooperation.

\section{Energetic Particle Detector}

The sensors of this instrument comprise two semiconductor detectors each, an anticoincidence scintillator to filter out the particles from lateral directions, and an electronic unit for data processing. The purpose of the experiment is to investigate the particles accelerated in the vicinity of the comet, superthermal ions and electrons emitted by the sun, and low-energy galactic cosmic radiation. The instrument will detect ions in the range between $20 \mathrm{keV}$ and $14 \mathrm{MeV}$ per nucleon. The telescope was prepared by a HungarianFRG-Soviet cooperation.

\section{Plasma Wave Detectors}

These instruments are designed to detect electrostatic and electromagnetic waves propagating in the plasma. The low-frequency wave detector measures waves between 0.1 and $1000 \mathrm{~Hz}$ with a sensitivity of $10 \mu \mathrm{V} / \mathrm{m}$. The high-frequency sensor measures electromagnetic waves in the $0-300-\mathrm{kHz}$ range with a sensitivity of about $3 \mu \mathrm{V} / \mathrm{m}$. The Langmuir probe included in the system determines the parameters characterizing the state of the cold comet plasma (temperature and density). These devices were prepared jointly by Czechoslovak, French, Polish, and Soviet experts.

\section{Magnetometers}

The flux-gate magnetometers measure the components of the magnetic field vector with an accuracy of $10^{-6}$ Gauss. They are located on a 5-m-long boom extending from the end of the solar cells. These devices were prepared by Austrian and Soviet experts.

Shortly after the VEGA encounter with Halley, measurements will also be made at the comet by the Giotto probe from the European Space Agency (ESA) and by Japan's first deep space probe, called Planet-A.

In addition to its own scientific program, VEGA will also help ESA to fine-tune the Giotto encounter with Halley on March 13, 1986. ESA is planning to have Giotto approach the nucleus to within about $500 \mathrm{~km}$. To do so, the ESA engineers need highly accurate information on the location and trajectory of the nucleus, information that can be provided by the VEGAs. Information of the location of the nucleus obtained from the VEGA television cameras will be given to the Giotto project in time to use it for Giotto's final course correction. Giotto's last preencounter maneuver will be performed 2 days before its fly-by and 5 days after the VEGA 1 encounter. Thus VEGA will act as a "pathfinder" for the European spacecraft.

Tamas I. Gombosi is presently a visiting scientist at the University of Michigan, on leave from the Central Research Institute for Physics, Budapest. He is currently engaged in time-dependent modeling of dusty cometary atmospheres and other planetary environments. He is a member

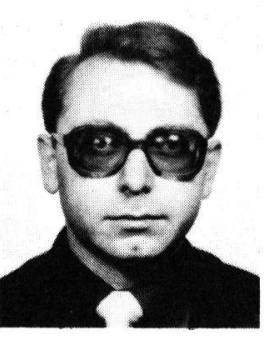
of the international VEGA science team. He graduated with an M.Sc. degree in physics from the Roland Eötvös University, Hungary, in 1970, and. received a Ph.D. in physics from the same university in 1974. He was awarded the academic degree of Candidate of Physical Sciences in 1979 and the degree of Doctor of Physical Sciences in 1983 by the Hungarian Academy of Sciences. He participated in the first significant measurement proving the existence of an $\sim 10^{14} \mathrm{eV}$ galactic cosmic ray anisotropy. In 1975-1976, while working at the Space Research Institute in Moscow, he took part in the first extensive plasma environment study of Venus. 